\title{
Spatio-temporal patterns of Pyrenean exhumation revealed by inverse thermo-kinematic modeling of a large thermochronologic dataset
}

Magdalena Ellis Curry¹, Peter van der Beek², Ritske S. Huismans ${ }^{3}$, Sebastian G. Wolf ${ }^{3}$, Charlotte Fillon ${ }^{4}$, Josep-Anton Muñoz

${ }^{1}$ University of Houston, Houston, Texas

${ }^{2}$ University of Potsdam, Potsdam, Germany

${ }^{3}$ University of Bergen, Bergen, Norway

${ }^{4}$ TOTAL Exploration Production, Pau, France

${ }^{5}$ University of Barcelona, Barcelona, Spain

\section{Data Repository}

\section{Table of Contents}

1.1 Thermo-kinematic modeling and inversion approach

1.2 Modeling of Miocene sediment blanketing

1.3 Erosion calculations

1.4 Uncertainty introduced by topographic scenario

Figure DR1: Inversion set up

Figure DR2: Plots of inversion results and uncertainties

Figure DR3: North-south trends of cooling ages

Figure DR4: Restored cross-sections through the western and eastern Pyrenees

Table DR1: Uncertainty calculation for topographic scenario

Table DR2: Thermochronology data compilation

Table DR3: Modeling parameters

\subsection{Thermo-kinematic modeling and inversion approach}

We utilize the thermo-kinematic modeling code Pecube to evaluate our hypotheses. Pecube uses a finite-element method to solve the heat transport equation in 3D (Braun, 2003). It then tracks the paths and temperatures of rocks as they are advected through a surface topography. Given a tectonic and topographic scenario together with crustal and thermal parameters, Pecube will predict cooling ages for any thermochronometer. Pecube tests tectonic scenarios by imposing spatially and temporally variable rock uplift and a topographic evolution scenario. Pecube incorporates the Neighborhood Algorithm (Sambridge, 1999a, 1999b), allowing for direct inversion of thermochronology data. The inversion approach allows identifying a best-fit tectonic and topographic scenario within a predefined parameter 
space. To evaluate the fit of the model, predicted thermochronological ages are compared to observed ages using a reduced Chi-square calculation:

$$
\mu=\sqrt{\frac{\sum_{i=1}^{n} \frac{\left(O_{i}-P_{i}\right)^{2}}{U_{i}^{2}}}{n}}
$$

Where misfit $(\mu)$ represents the difference between observed $\left(O_{i}\right)$ and predicted $\left(P_{i}\right)$ thermochronologic ages, and incorporates published uncertainty on individual ages $\left(U_{i}\right) ; n$ is the number of data. Each model run represents 10,080 forward models that converge on a combination of input parameters with the lowest calculated misfit. We then assess the inversion results by calculating the marginal posterior probability density function (PDF) of each inverted parameter, thus providing a Bayesian estimate of parameter resolution (Sambridge, 1999b; Valla et al., 2010; Glotzbach et al., 2011; Fillon and van der Beek, 2012).

\subsection{Modeling of Miocene sediment blanketing (Figure DR1)}

To incorporate the effects of Miocene sediment blanketing on thermochronometric ages we modify the source code to impose a minimum elevation in the same manner as Fillon and van der Beek (2012). We allow the minimum elevation to increase linearly between 34 and $23 \mathrm{Ma}$, simulating deposition in the valleys. The minimum elevation starts at $0 \mathrm{~km}$ at $34 \mathrm{Ma}$, and we invert for the thickness at $23 \mathrm{Ma}$ and 10 Ma. The thicknesses at $23 \mathrm{Ma}$ and $10 \mathrm{Ma}$ are inverted separately, thus allowing for different values at these two times, searching between $0-2.5 \mathrm{~km}$ for the best fit. From $10 \mathrm{Ma}$ to present, the minimum elevation decreases linearly back to $0 \mathrm{~km}$, simulating incision. The sediment added has the same thermal properties as the modeled crust and is compensated with flexural isostasy.

\subsection{Erosion calculations}

To calculate eroded material through time, we combine the topographic evolution proposed by Curry et al. (2019) with our best-fit results from the suite of inversions, which provide rock uplift through time. If there was no topographic change (i.e. in topographic steady state), rock uplift (Ur) simply equals erosion (E). With topographic change $\Delta$ Topo (positive in case of topographic growth), Ur $=\mathrm{E}+\Delta \mathrm{Topo}$ (e.g., England and Molnar, 1990). 
We calculate the rock uplift for each cell $\left(\sim 700 \mathrm{~m}^{2}\right)$ for each time bin of the inversion, and subtract the predicted topographic change. In this way we get a value for the amount of erosion for each cell at each time bin. To calculate the cumulative 1D erosion (Figure 3B, C in main text), we sum the cumulative erosion through time for each cell and report the mean value of erosion within the zone of interest.

\subsection{Uncertainty introduced by topographic scenario}

We use the first order topographic evolution proposed by Curry et al. (2019) to constrain the surface boundary conditions of our model (see Fig. DR1 below). Curry et al. (2019) used flexural modeling and basin reconstructions to estimate the change in the Pyrenean topographic load through time. The uncertainty this scenario introduces into our inferred rock uplift and exhumation rate is $\pm 0.007-0.038$ $\mathrm{km} / \mathrm{m}$.y., with the highest uncertainty $(0.038 \mathrm{~km} / \mathrm{m} . \mathrm{y}$.) during the late Eocene when there was rapid growth of topography (Table DR1, below). This uncertainty is calculated by propagating the uncertainty in surface uplift rate (in $\mathrm{km} / \mathrm{m} . \mathrm{y}$.) onto our results, following again the simple equation $\mathrm{Ur}=\mathrm{E}+\Delta \mathrm{Topo}$ (England and Molnar, 1990).

Table DR1: Calculation of uncertainty introduced by topographic scenario

\begin{tabular}{|c|c|c|c|}
\hline Time (Ma) & $\begin{array}{c}\text { Tau value from Curry } \\
\text { et al. (2019) } \\
\text { [proportion of modern } \\
\text { topography] }\end{array}$ & $\begin{array}{c}\text { Mean } \\
\text { topographic } \\
\text { elevation } \\
\text { through time } \\
(\mathrm{m})\end{array}$ & $\begin{array}{c}\text { Uncertainty on } \\
\text { mean } \\
\text { topography } \\
(\mathrm{m})^{* *}\end{array}$ \\
\hline 0 & 1 & 1469 & 150 \\
\hline 23 & 1.1 & 1763 & 150 \\
\hline 34 & 1 & 1396 & 150 \\
\hline 38 & 0.8 & 1028 & 150 \\
\hline 56 & 0.6 & 881 & 150 \\
\hline
\end{tabular}

\begin{tabular}{|c|c|c|c|c|c|c|}
\hline $\begin{array}{c}\text { Time span } \\
\text { (Ma) }\end{array}$ & $\begin{array}{l}\text { Time } \\
\text { (m.y.) }\end{array}$ & $\begin{array}{c}\text { Delta mean } \\
(\mathrm{m})\end{array}$ & $\begin{array}{c}\text { Mean rate } \\
(\mathrm{m} / \mathrm{my})\end{array}$ & $\begin{array}{c}\text { Tau } 0.1 \text { rate } \\
\text { (m/m.y.) }\end{array}$ & $\begin{array}{c}\text { Mean rate of } \\
\text { topographic change } \\
(\mathrm{km} / \mathrm{m} . \mathrm{y} .)^{* *} \text { Used } \\
\text { in model. }\end{array}$ & $\begin{array}{c}\text { Uncertainty in } \\
\text { topographic } \\
\text { scenario } \\
\text { (km/m.y.) }\end{array}$ \\
\hline $23-0$ & 23 & -294 & -12.8 & 6.5 & -0.013 & 0.007 \\
\hline $34-23$ & 11 & 367 & 33.4 & 13.6 & 0.033 & 0.014 \\
\hline 38-34 & 4 & 367 & 91.8 & 37.5 & 0.092 & 0.038 \\
\hline $56-38$ & 18 & 147 & 8.2 & 8.3 & 0.008 & 0.008 \\
\hline
\end{tabular}

** Uncertainty from Curry et al. (2019) is represented as 0.1 of Tau, which is a proportion of modern topography. The modern mean topography is 1469 in our study area, we use plus/minus 150 meters 

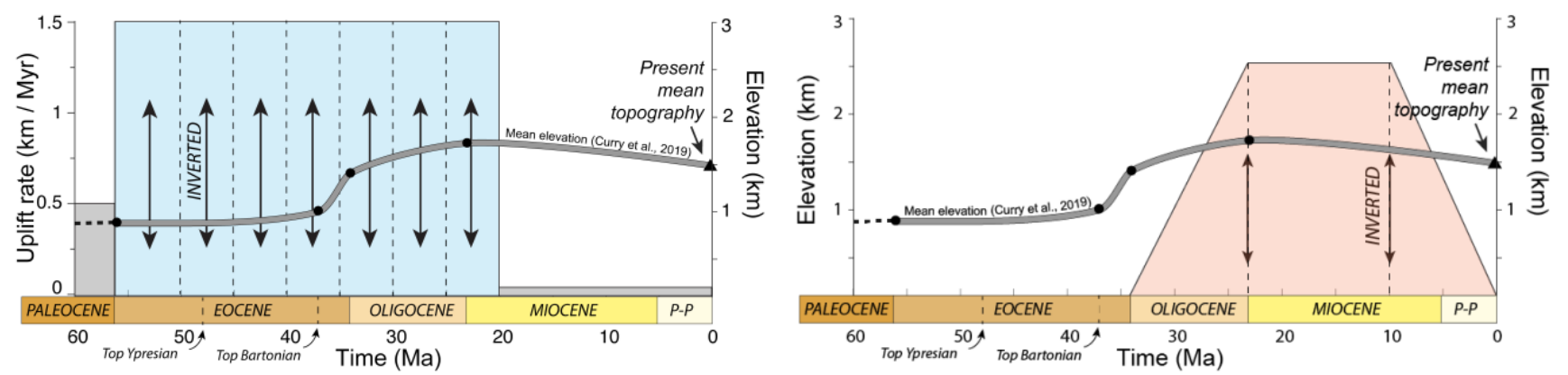

Figure DR1: Inversion set up. A) Time bins within which we invert for uplift rate, and range of values the inversion searches within. B) Sediment blanketing: Miocene sediment imposed (34-23 Ma) and removed (10-0 Ma) to simulate deposition and erosion of sediment in valleys. Gray line is mean swath elevation of the Pyrenees based on results of Curry et al. (2019). Double arrows indicate parameters that are inverted for.
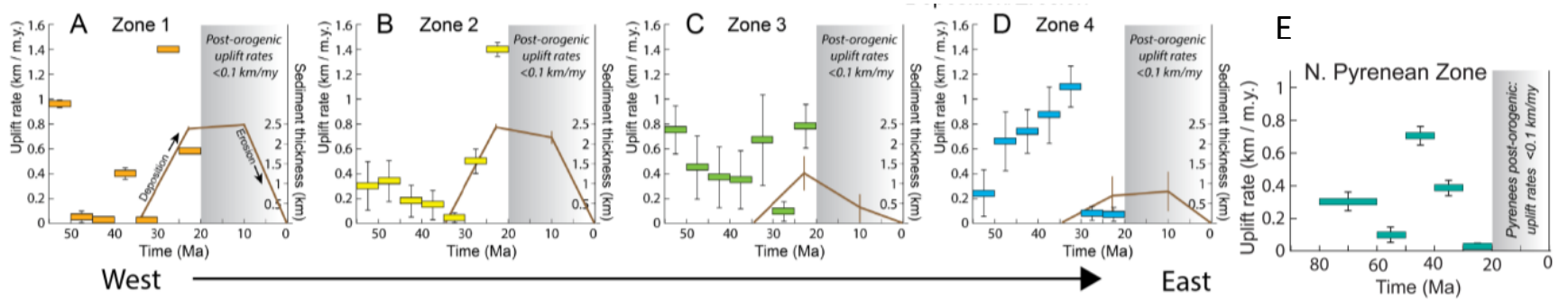

Figure DR2: Inversion results and uncertainties for best-fit model B (A-D) and North Pyrenean Zone (E). Inversion results for uplift rate through time, and thickness of sediment blanketing (brown lines in A-D, details in section DR1.2), organized by zone. Uncertainty bars correspond to the standard deviation of the marginal posterior probability density function of each inverted parameter, which provides a Bayesian estimate of parameter resolution. 

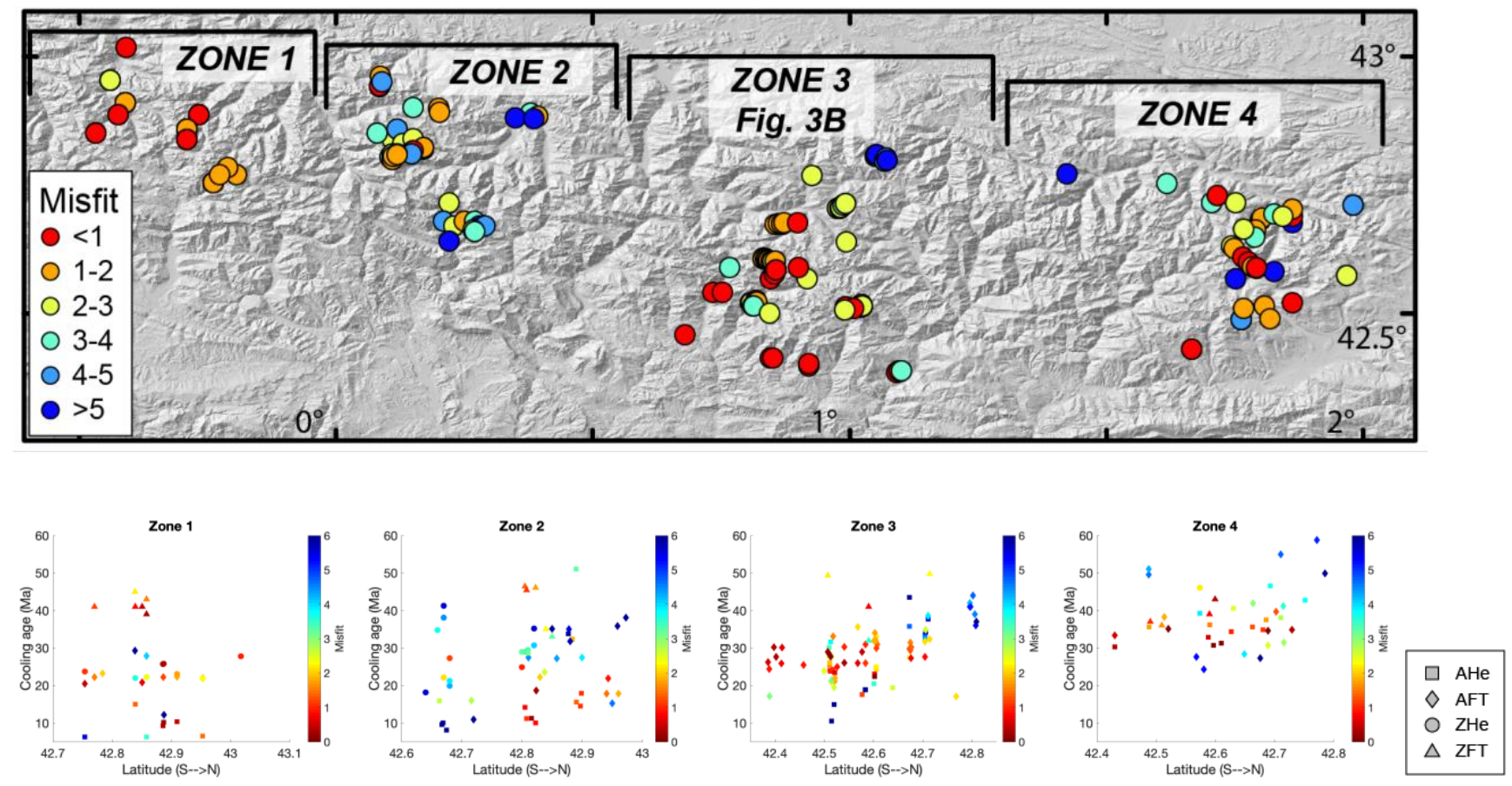

Figure DR3: Map of misfit and plots of north-south pattern of cooling ages for model B, separated by E$\mathrm{W}$ zone and colored by misfit, symbols indicate different thermochronometers. Note the southwardyounging ages in Zone 3 mentioned in the text, but lack of southward younging in other zones. 


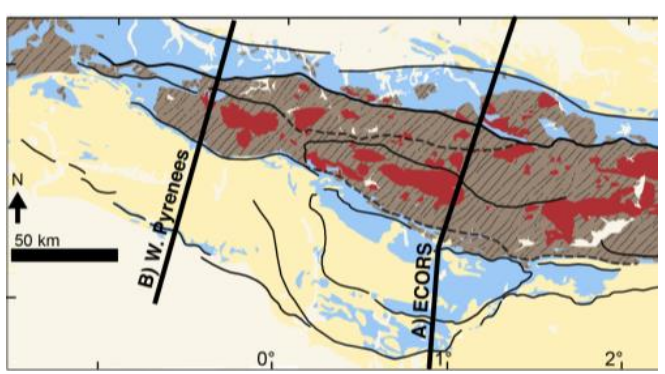

A) Eastern Pyrenees (ECORS) restoration Pre-convergence

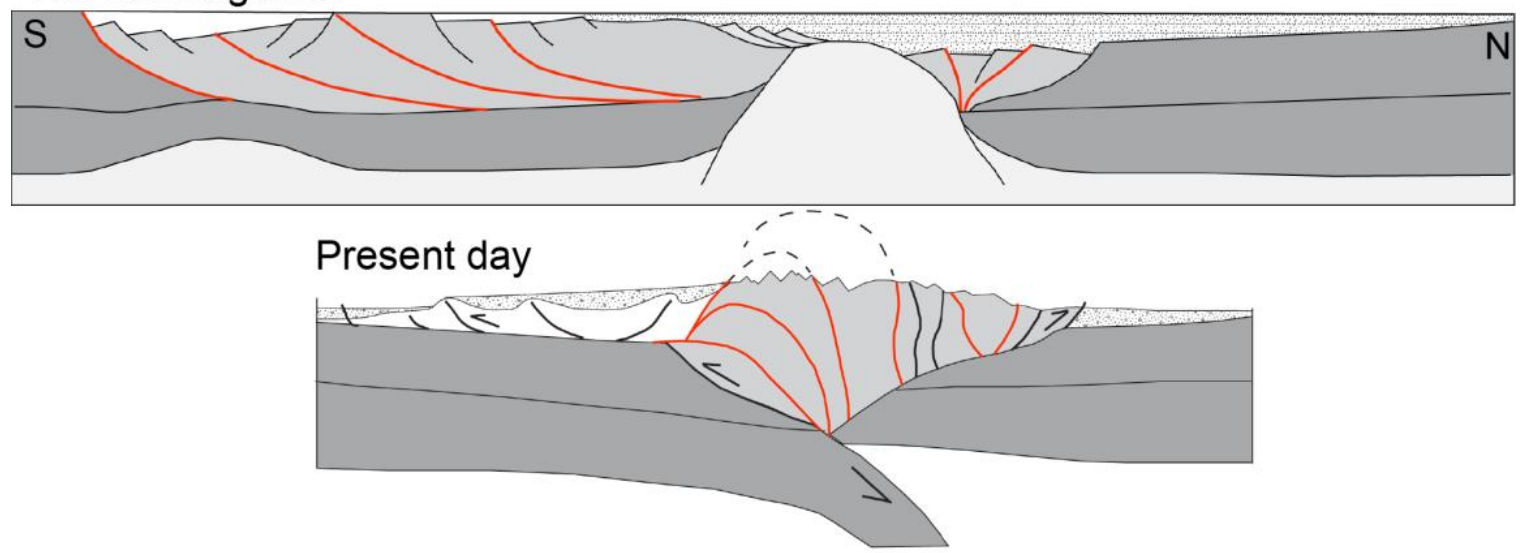

B) Western Pyrenees restoration

\section{Pre-convergence}

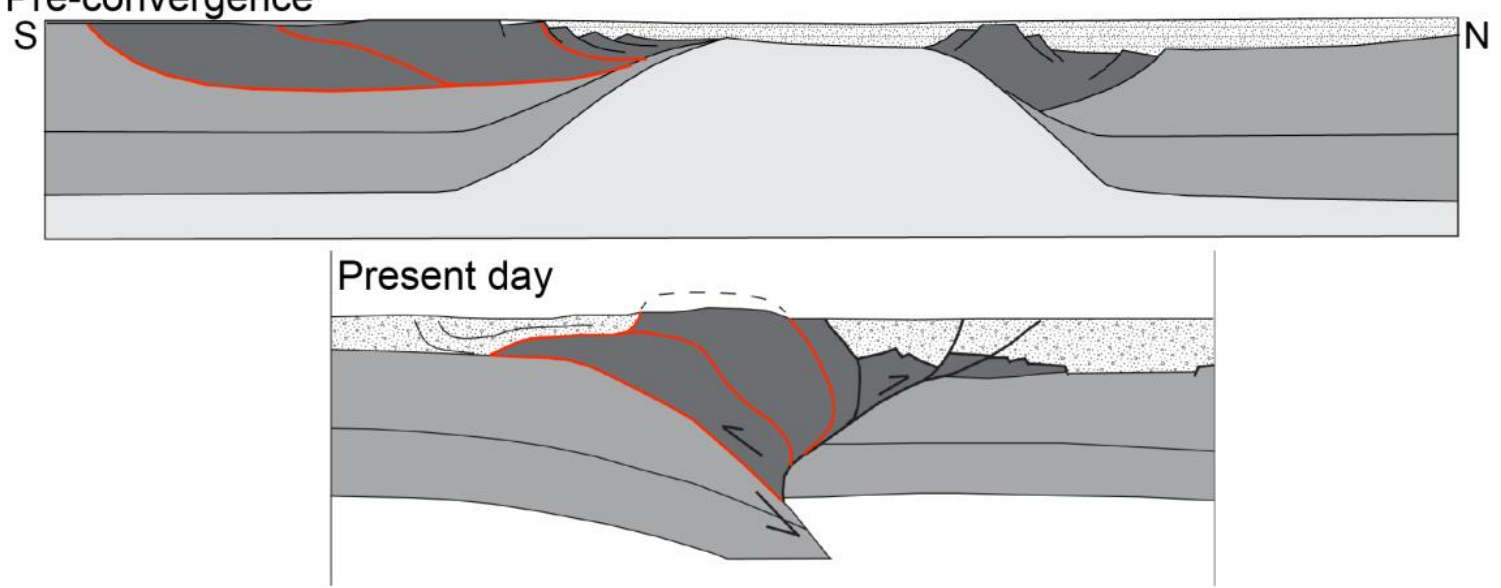

Figure DR4: Restored cross-sections and location map from A) eastern (our Zone 3) and B) western (our Zone 1) Pyrenees. Modified from Muñoz (1992), Sinclair et al. (2005), Jammes et al. (2014), Teixell et al. (2016). 
Table DR2: Thermochronology data compilation

\begin{tabular}{|c|c|c|c|c|c|c|c|c|c|c|c|c|c|c|c|}
\hline Longitude & Latitude & $\begin{array}{l}\text { Elevation } \\
\text { (meters) }\end{array}$ & $\mathrm{AHe}$ & $\begin{array}{l}\mathrm{AHe} \\
\text { error }\end{array}$ & AFT & $\begin{array}{l}\text { AFT } \\
\text { error }\end{array}$ & $\mathrm{ZHe}$ & $\begin{array}{l}\mathrm{ZHe} \\
\text { error }\end{array}$ & ZFT & $\begin{array}{l}\text { ZFT } \\
\text { error }\end{array}$ & $\begin{array}{c}\text { EW } \\
\text { Zone }\end{array}$ & Region & Sample type* & $\begin{array}{l}\text { \# Samples / } \\
\text { \# Grains }\end{array}$ & Reference \\
\hline-0.44 & 42.953 & 677 & 6.6 & 0.8 & & & & & & & Zone 1 & Axial Zone & M-S & $3 / 4$ & Bosch et al., 2016 \\
\hline-0.424 & 42.887 & 1066 & 10 & 6.1 & & & & & & & Zone 1 & Axial Zone & $M-M$ & $4 / 6$ & Bosch et al., 2016 \\
\hline-0.41 & 42.91 & 1208 & 10 & 3.0 & & & & & & & Zone 1 & Axial Zone & $M-M$ & $4 / 4$ & Bosch et al., 2016 \\
\hline-0.29 & 42.839 & 3147 & 15 & 3.4 & & & & & & & Zone 1 & Axial Zone & $M-M$ & $2 / 5$ & Bosch et al., 2016 \\
\hline-0.29 & 42.858 & 2080 & 6.3 & 1.0 & & & & & & & Zone 1 & Axial Zone & $M-M$ & $2 / 4$ & Bosch et al., 2016 \\
\hline-0.267 & 42.886 & 1417 & 9.3 & 5.6 & & & & & & & Zone 1 & Axial Zone & $M-M$ & $4 / 4$ & Bosch et al., 2016 \\
\hline-0.239 & 42.754 & 1614 & 6.3 & 0.5 & & & & & & & Zone 1 & Axial Zone & $M-M$ & $3 / 7$ & Bosch et al., 2016 \\
\hline 0.106 & 42.804 & 2879 & 29 & 3.6 & & & & & & & Zone 2 & Axial Zone & S-M & $1 / 2$ & Fillon et al., in review \\
\hline 0.111 & 42.805 & 2707 & 14 & 2.6 & & & & & & & Zone 2 & Axial Zone & S-M & $1 / 6$ & Fillon et al., in review \\
\hline 0.119 & 42.808 & 2423 & 11 & 0.9 & & & & & & & Zone 2 & Axial Zone & S-M & $1 / 2$ & Fillon et al., in review \\
\hline 0.151 & 42.816 & 2038 & 11 & 1.5 & & & & & & & Zone 2 & Axial Zone & S-M & $1 / 2$ & Fillon et al., in review \\
\hline 0.170 & 42.823 & 1864 & 10 & 1.8 & & & & & & & Zone 2 & Axial Zone & S-M & $1 / 7$ & Fillon et al., in review \\
\hline 0.200 & 42.897 & 2032 & 15 & 3.0 & & & & & & & Zone 2 & Axial Zone & S-M & $1 / 3$ & Fillon et al., in review \\
\hline 0.201 & 42.899 & 2013 & 18 & 6.2 & & & & & & & Zone 2 & Axial Zone & S-M & $1 / 4$ & Fillon et al., in review \\
\hline 0.202 & 42.891 & 2135 & 16 & 2.9 & & & & & & & Zone 2 & Axial Zone & S-M & $1 / 3$ & Fillon et al., in review \\
\hline 0.247 & 42.675 & 1532 & 8.1 & 1.0 & & & & & & & Zone 2 & Axial Zone & S-M & $1 / 7$ & Fillon et al., in review \\
\hline 0.267 & 42.663 & 2528 & 16 & 1.3 & & & & & & & Zone 2 & Axial Zone & S-M & $1 / 2$ & Fillon et al., in review \\
\hline 0.28 & 42.669 & 2221 & 10 & 1.4 & & & & & & & Zone 2 & Axial Zone & S-M & $1 / 2$ & Fillon et al., in review \\
\hline 0.285 & 42.667 & 2209 & 9.6 & 1.7 & & & & & & & Zone 2 & Axial Zone & S-M & $1 / 2$ & Fillon et al., in review \\
\hline 0.368 & 42.877 & 1100 & 34 & 2.7 & & & & & & & Zone 2 & Axial Zone & S-S & $1 / 1$ & Fillon et al., in review \\
\hline 0.379 & 42.890 & 779 & 51 & 12.1 & & & & & & & Zone 2 & Axial Zone & S-M & $1 / 3$ & Fillon et al., in review \\
\hline 0.393 & 42.884 & 1209 & 32 & 10.9 & & & & & & & Zone 2 & Axial Zone & S-M & $1 / 2$ & Fillon et al., in review \\
\hline 0.832 & 42.606 & 2735 & 24 & 1.4 & & & & & & & Zone 3 & Axial Zone & S-M & $1 / 3$ & Metcalf et al., 2009 \\
\hline 0.836 & 42.606 & 2605 & 25 & 1.5 & & & & & & & Zone 3 & Axial Zone & S-M & $1 / 3$ & Metcalf et al., 2009 \\
\hline 0.84 & 42.605 & 2495 & 25 & 1.4 & & & & & & & Zone 3 & Axial Zone & S-M & $1 / 3$ & Metcalf et al., 2009 \\
\hline 0.847 & 42.602 & 2210 & 23 & 1.4 & & & & & & & Zone 3 & Axial Zone & S-M & $1 / 3$ & Metcalf et al., 2009 \\
\hline 0.85 & 42.601 & 2080 & 21 & 0.5 & & & & & & & Zone 3 & Axial Zone & $M-M$ & $2 / 6$ & Metcalf et al., 2009 \\
\hline 0.852 & 42.673 & 2090 & 44 & 3.5 & & & & & & & Zone 3 & Axial Zone & S-M & $1 / 3$ & Metcalf et al., 2009 \\
\hline 0.853 & 42.577 & 1620 & 18 & 3.9 & & & & & & & Zone 3 & Axial Zone & $M-M$ & $3 / 5$ & Metcalf et al., 2009 \\
\hline 0.855 & 42.602 & 1945 & 22 & 1.4 & & & & & & & Zone 3 & Axial Zone & S-M & $1 / 3$ & Metcalf et al., 2009 \\
\hline 0.856 & 42.583 & 1680 & 19 & 0.5 & & & & & & & Zone 3 & Axial Zone & $M-M$ & $2 / 6$ & Metcalf et al., 2009 \\
\hline 0.857 & 42.584 & 1780 & 19 & 0.4 & & & & & & & Zone 3 & Axial Zone & $M-M$ & $2 / 6$ & Metcalf et al., 2009 \\
\hline 0.994 & 42.639 & 2120 & 19 & 1.3 & & & & & & & Zone 3 & Axial Zone & $M-M$ & $2 / 6$ & Metcalf et al., 2009 \\
\hline 0.868 & 42.673 & 1480 & 28 & 2.2 & & & & & & & Zone 3 & Axial Zone & S-M & $1 / 5$ & Gibson et al., 2007 \\
\hline 0.869 & 42.673 & 1415 & 36 & 2.9 & & & & & & & Zone 3 & Axial Zone & $M-M$ & $2 / 11$ & Gibson, 2005 (Thesis) \\
\hline 0.976 & 42.705 & 2030 & 34 & 2.7 & & & & & & & Zone 3 & Axial Zone & $M-M$ & $3 / 21$ & Gibson et al., 2007 \\
\hline 0.981 & 42.705 & 2200 & 32 & 2.6 & & & & & & & Zone 3 & Axial Zone & S-M & $1 / 5$ & Gibson et al., 2007 \\
\hline 0.983 & 42.706 & 2304 & 34 & 2.3 & & & & & & & Zone 3 & Axial Zone & S-M & $1 / 7$ & Gibson et al., 2007 \\
\hline 0.988 & 42.711 & 2440 & 38 & 3.0 & & & & & & & Zone 3 & Axial Zone & S-M & $1 / 6$ & Gibson et al., 2007 \\
\hline 0.993 & 42.513 & 2030 & 24 & 1.9 & & & & & & & Zone 3 & Axial Zone & $M-M$ & $2 / 26$ & Gibson et al., 2007 \\
\hline 0.994 & 42.511 & 1920 & 25 & 2.0 & & & & & & & Zone 3 & Axial Zone & $\mathrm{M}-\mathrm{M}$ & $2 / 25$ & Gibson et al., 2007 \\
\hline 1.001 & 42.51 & 2140 & 30 & 2.4 & & & & & & & Zone 3 & Axial Zone & S-M & $1 / 8$ & Gibson et al., 2007 \\
\hline 1.008 & 42.51 & 2250 & 28 & 2.3 & & & & & & & Zone 3 & Axial Zone & $M-M$ & $2 / 14$ & Gibson et al., 2007 \\
\hline 1.013 & 42.512 & 2360 & 24 & 2.0 & & & & & & & Zone 3 & Axial Zone & S-M & $1 / 5$ & Gibson et al., 2007 \\
\hline 1.02 & 42.511 & 2440 & 26 & 2.0 & & & & & & & Zone 3 & Axial Zone & S-M & $1 / 8$ & Gibson et al., 2007 \\
\hline 1.024 & 42.518 & 2870 & 24 & 1.9 & & & & & & & Zone 3 & Axial Zone & S-M & $3 / 20$ & Gibson et al., 2007 \\
\hline 1.025 & 42.513 & 2650 & 32 & 2.5 & & & & & & & Zone 3 & Axial Zone & $\mathrm{M}-\mathrm{M}$ & $3 / 32$ & Gibson et al., 2007 \\
\hline 1.025 & 42.514 & 2765 & 28 & 2.2 & & & & & & & Zone 3 & Axial Zone & $M-M$ & $2 / 13$ & Gibson et al., 2007 \\
\hline 0.807 & 42.521 & 1750 & 23 & 2.0 & & & & & & & Zone 3 & Axial Zone & $M-M$ & $3 / 23$ & Gibson et al., 2007 \\
\hline 0.808 & 42.52 & 1695 & 15 & 1.2 & & & & & & & Zone 3 & Axial Zone & $M-M$ & $2 / 23$ & Gibson et al., 2007 \\
\hline 0.813 & 42.515 & 1150 & 11 & 1.0 & & & & & & & Zone 3 & Axial Zone & S-M & $1 / 10$ & Gibson et al., 2007 \\
\hline 1.618 & 42.752 & 1850 & 43 & 3.0 & & & & & & & Zone 4 & Axial Zone & $M-M$ & $3 / 9$ & Gunnel et al., 2009 \\
\hline 1.666 & 42.43 & 1945 & 30 & 2.1 & & & & & & & Zone 4 & Axial Zone & $M-M$ & $4 / 8$ & Gunnel et al., 2009 \\
\hline 1.98 & 42.711 & 2190 & 38 & 2.6 & & & & & & & Zone 4 & Axial Zone & $\mathrm{M}-\mathrm{M}$ & $3 / 9$ & Gunnel et al., 2009 \\
\hline 1.763 & 42.488 & 2900 & 35 & 2.5 & & & & & & & Zone 4 & Axial Zone & $M-M$ & $2 / 4$ & Gunnel et al., 2009 \\
\hline 1.744 & 42.631 & 2580 & 41 & 3.7 & & & & & & & Zone 4 & Axial Zone & $M-M$ & $2 / 20$ & Denèle, 2007 (PhD Thesis) \\
\hline 1.748 & 42.627 & 2783 & 34 & 3.1 & & & & & & & Zone 4 & Axial Zone & S-M & $1 / 10$ & Denèle, 2007 (PhD Thesis) \\
\hline 1.766 & 42.61 & 2210 & 31 & 2.8 & & & & & & & Zone 4 & Axial Zone & $M-M$ & $2 / 20$ & Denèle, 2007 (PhD Thesis) \\
\hline 1.777 & 42.597 & 2000 & 31 & 2.8 & & & & & & & Zone 4 & Axial Zone & S-M & $1 / 10$ & Denèle, 2007 (PhD Thesis) \\
\hline 1.785 & 42.591 & 1750 & 36 & 3.3 & & & & & & & Zone 4 & Axial Zone & $\mathrm{M}-\mathrm{M}$ & $2 / 20$ & Denèle, 2007 (PhD Thesis) \\
\hline 1.79 & 42.663 & 2250 & 36 & 3.2 & & & & & & & Zone 4 & Axial Zone & S-M & $1 / 10$ & Denèle, 2007 (PhD Thesis) \\
\hline
\end{tabular}

* S-S = Single sample, single grain; S-M = Single sample, multiple grain; $M-M=$ Multiple sample, multiple grain; M-S = Multiple sample, single grain; NR = Not Reported 


\begin{tabular}{|c|c|c|c|c|c|c|c|c|c|c|c|c|c|c|c|}
\hline Longitude & Latitude & $\begin{array}{l}\text { Elevation } \\
\text { (meters) }\end{array}$ & $\mathrm{AHe}$ & $\begin{array}{l}\text { AHe } \\
\text { error }\end{array}$ & AFT & $\begin{array}{l}\text { AFT } \\
\text { error }\end{array}$ & $\mathrm{ZHe}$ & $\begin{array}{l}\text { ZHe } \\
\text { error }\end{array}$ & ZFT & $\begin{array}{c}\begin{array}{c}\text { ZFT } \\
\text { error }\end{array} \\
\end{array}$ & $\begin{array}{c}\text { EW } \\
\text { Zone }\end{array}$ & Region & Sample type* & $\begin{array}{l}\text { \# Samples / } \\
\text { \# Grains }\end{array}$ & Reference \\
\hline 1.792 & 42.588 & 1450 & 33 & 3.0 & & & & & & & Zone 4 & Axial Zone & $M-M$ & $2 / 20$ & Denèle, 2007 (PhD Thesis) \\
\hline 1.8 & 42.681 & 1800 & 35 & 3.2 & & & & & & & Zone 4 & Axial Zone & $M-M$ & $2 / 20$ & Denèle, 2007 (PhD Thesis) \\
\hline 1.803 & 42.686 & 1550 & 38 & 3.4 & & & & & & & Zone 4 & Axial Zone & S-M & $1 / 10$ & Denèle, 2007 (PhD Thesis) \\
\hline 1.827 & 42.693 & 900 & 47 & 4.2 & & & & & & & Zone 4 & Axial Zone & $M-M$ & $2 / 20$ & Denèle, 2007 (PhD Thesis) \\
\hline 1.968 & 42.574 & 2320 & 39 & 2.0 & & & & & & & Zone 4 & Axial Zone & $M-M$ & $2 / 20$ & Maurel et al., 2008 \\
\hline-0.267 & 42.886 & 1417 & & & 22 & 1.1 & & & & & Zone 1 & Axial Zone & S-M & $1 / 24$ & Bosch et al., 2016 \\
\hline-0.290 & 42.839 & 3137 & & & 29 & 1.4 & & & & & Zone 1 & Axial Zone & S-M & $1 / 20$ & Bosch et al., 2016 \\
\hline-0.290 & 42.858 & 2080 & & & 28 & 1.8 & & & & & Zone 1 & Axial Zone & S-M & $1 / 20$ & Bosch et al., 2016 \\
\hline-0.239 & 42.754 & 1614 & & & 21 & 0.9 & & & & & Zone 1 & Axial Zone & S-M & $1 / 20$ & Bosch et al., 2016 \\
\hline-0.424 & 42.887 & 1066 & & & 12 & 1.1 & & & & & Zone 1 & Axial Zone & S-M & $1 / 15$ & Bosch et al., 2016 \\
\hline-0.410 & 42.910 & 1208 & & & 22 & 1.2 & & & & & Zone 1 & Axial Zone & S-M & $1 / 22$ & Bosch et al., 2016 \\
\hline-0.440 & 42.953 & 677 & & & 22 & 1.6 & & & & & Zone 1 & Axial Zone & S-M & $1 / 13$ & Bosch et al., 2016 \\
\hline 0.085 & 42.941 & 1838 & & & 18 & 1.5 & & & & & Zone 2 & Axial Zone & S-M & $1 / 8$ & Mouchene, 2016 (PhD thesis) \\
\hline 0.086 & 42.943 & 1669 & & & 22 & 2.1 & & & & & Zone 2 & Axial Zone & S-M & $1 / 15$ & Mouchene, 2016 (PhD thesis) \\
\hline 0.087 & 42.961 & 1082 & & & 18 & 1.2 & & & & & Zone 2 & Axial Zone & S-M & $1 / 20$ & Mouchene, 2016 (PhD thesis) \\
\hline 0.090 & 42.950 & 1420 & & & 15 & 1.0 & & & & & Zone 2 & Axial Zone & S-M & $1 / 20$ & Mouchene, 2016 (PhD thesis) \\
\hline 0.35 & 42.88 & 900 & & & 32 & 2.2 & & & & & Zone 2 & Axial Zone & S-M & $1 / 20$ & Jolivet et al., 2007 \\
\hline 0.08 & 42.85 & 3100 & & & 35 & 2.3 & & & & & Zone 2 & Axial Zone & S-M & $1 / 20$ & Jolivet et al., 2007 \\
\hline 0.13 & 42.83 & 1900 & & & 22 & 1.4 & & & & & Zone 2 & Axial Zone & S-M & $1 / 20$ & Jolivet et al., 2007 \\
\hline 0.15 & 42.9 & 2100 & & & 28 & 2.1 & & & & & Zone 2 & Axial Zone & S-M & $1 / 20$ & Jolivet et al., 2007 \\
\hline 0.18 & 42.72 & 1400 & & & 11 & 1.1 & & & & & Zone 2 & Axial Zone & S-M & $1 / 20$ & Jolivet et al., 2007 \\
\hline 0.976 & 42.705 & 2030 & & & 32 & 2.8 & & & & & Zone 3 & Axial Zone & S-M & $1 / 24$ & Sinclair et al., 2005 \\
\hline 0.981 & 42.705 & 2200 & & & 28 & 2.4 & & & & & Zone 3 & Axial Zone & S-M & $1 / 25$ & Sinclair et al., 2005 \\
\hline 0.983 & 42.706 & 2304 & & & 35 & 3.6 & & & & & Zone 3 & Axial Zone & S-M & $1 / 24$ & Sinclair et al., 2005 \\
\hline 0.988 & 42.711 & 2440 & & & 39 & 3.2 & & & & & Zone 3 & Axial Zone & S-M & $1 / 25$ & Sinclair et al., 2005 \\
\hline 0.99 & 42.507 & 1750 & & & 29 & 3.1 & & & & & Zone 3 & Axial Zone & S-M & $1 / 24$ & Sinclair et al., 2005 \\
\hline 0.993 & 42.714 & 2635 & & & 32 & 2.8 & & & & & Zone 3 & Axial Zone & S-M & $1 / 20$ & Sinclair et al., 2005 \\
\hline 0.993 & 42.513 & 2030 & & & 28 & 2.5 & & & & & Zone 3 & Axial Zone & S-M & $1 / 25$ & Sinclair et al., 2005 \\
\hline 1.008 & 42.51 & 2250 & & & 29 & 3.0 & & & & & Zone 3 & Axial Zone & S-M & $1 / 25$ & Sinclair et al., 2005 \\
\hline 1.024 & 42.518 & 2870 & & & 33 & 3.4 & & & & & Zone 3 & Axial Zone & S-M & $1 / 25$ & Sinclair et al., 2005 \\
\hline 0.807 & 42.521 & 1780 & & & 21 & 3.7 & & & & & Zone 3 & Axial Zone & S-M & $1 / 17$ & Sinclair et al., 2005 \\
\hline 0.808 & 42.52 & 1695 & & & 20 & 3.2 & & & & & Zone 3 & Axial Zone & S-M & $1 / 17$ & Sinclair et al., 2005 \\
\hline 0.813 & 42.515 & 1150 & & & 21 & 2.0 & & & & & Zone 3 & Axial Zone & S-M & $1 / 24$ & Sinclair et al., 2005 \\
\hline 1.092 & 42.386 & 1280 & & & 26 & 3.1 & & & & & Zone 3 & Axial Zone & S-M & $1 / 25$ & Sinclair et al., 2005 \\
\hline 1.097 & 42.388 & 1085 & & & 24 & 3.9 & & & & & Zone 3 & Axial Zone & S-M & $1 / 25$ & Sinclair et al., 2005 \\
\hline 1.101 & 42.389 & 960 & & & 17 & 3.4 & & & & & Zone 3 & Axial Zone & S-M & $1 / 9$ & Sinclair et al., 2005 \\
\hline 0.832 & 42.608 & 2850 & & & 31 & 3.0 & & & & & Zone 3 & Axial Zone & S-M & $1 / 19$ & Fitzgerald et al., 1999 \\
\hline 0.832 & 42.606 & 2735 & & & 30 & 3.0 & & & & & Zone 3 & Axial Zone & S-M & $1 / 20$ & Fitzgerald et al., 1999 \\
\hline 0.836 & 42.606 & 2605 & & & 33 & 3.0 & & & & & Zone 3 & Axial Zone & S-M & $1 / 20$ & Fitzgerald et al., 1999 \\
\hline 0.84 & 42.605 & 2495 & & & 32 & 3.0 & & & & & Zone 3 & Axial Zone & S-M & $1 / 22$ & Fitzgerald et al., 1999 \\
\hline 0.843 & 42.603 & 2355 & & & 34 & 4.0 & & & & & Zone 3 & Axial Zone & S-M & $1 / 9$ & Fitzgerald et al., 1999 \\
\hline 0.846 & 42.568 & 1500 & & & 26 & 3.0 & & & & & Zone 3 & Axial Zone & S-M & $1 / 19$ & Fitzgerald et al., 1999 \\
\hline 0.847 & 42.602 & 2210 & & & 32 & 3.0 & & & & & Zone 3 & Axial Zone & S-M & $1 / 20$ & Fitzgerald et al., 1999 \\
\hline 0.851 & 42.601 & 2080 & & & 32 & 2.0 & & & & & Zone 3 & Axial Zone & S-M & $1 / 21$ & Fitzgerald et al., 1999 \\
\hline 0.853 & 42.577 & 1620 & & & 29 & 3.0 & & & & & Zone 3 & Axial Zone & S-M & $1 / 20$ & Fitzgerald et al., 1999 \\
\hline 0.855 & 42.602 & 1945 & & & 32 & 2.0 & & & & & Zone 3 & Axial Zone & S-M & $1 / 20$ & Fitzgerald et al., 1999 \\
\hline 0.857 & 42.584 & 1400 & & & 26 & 2.0 & & & & & Zone 3 & Axial Zone & S-M & $1 / 20$ & Fitzgerald et al., 1999 \\
\hline 0.857 & 42.584 & 1780 & & & 31 & 3.0 & & & & & Zone 3 & Axial Zone & S-M & $1 / 20$ & Fitzgerald et al., 1999 \\
\hline 1.05 & 42.806 & 1340 & & & 39 & 3.0 & & & & & Zone 3 & Axial Zone & S-M & $1 / 25$ & Fitzgerald et al., 1999 \\
\hline 1.052 & 42.808 & 1460 & & & 36 & 2.0 & & & & & Zone 3 & Axial Zone & S-M & $1 / 20$ & Fitzgerald et al., 1999 \\
\hline 1.053 & 42.809 & 1595 & & & 37 & 2.0 & & & & & Zone 3 & Axial Zone & S-M & $1 / 25$ & Fitzgerald et al., 1999 \\
\hline 1.068 & 42.796 & 2050 & & & 41 & 3.0 & & & & & Zone 3 & Axial Zone & S-M & $1 / 25$ & Fitzgerald et al., 1999 \\
\hline 1.069 & 42.796 & 2205 & & & 42 & 4.0 & & & & & Zone 3 & Axial Zone & S-M & $1 / 20$ & Fitzgerald et al., 1999 \\
\hline 1.072 & 42.802 & 2483 & & & 44 & 4.0 & & & & & Zone 3 & Axial Zone & S-M & $1 / 20$ & Fitzgerald et al., 1999 \\
\hline 1.073 & 42.797 & 2340 & & & 41 & 3.0 & & & & & Zone 3 & Axial Zone & S-M & $1 / 18$ & Fitzgerald et al., 1999 \\
\hline 0.812 & 42.514 & 1125 & & & 21 & 3.0 & & & & & Zone 3 & Axial Zone & S-M & $1 / 5$ & Fitzgerald et al., 1999 \\
\hline 0.818 & 42.526 & 1365 & & & 25 & 3.0 & & & & & Zone 3 & Axial Zone & S-M & $1 / 20$ & Fitzgerald et al., 1999 \\
\hline 0.819 & 42.522 & 1255 & & & 22 & 3.0 & & & & & Zone 3 & Axial Zone & S-M & $1 / 19$ & Fitzgerald et al., 1999 \\
\hline 0.855 & 42.673 & 2164 & & & 30 & 2.1 & & & & & Zone 3 & Axial Zone & S-M & $1 / 24$ & Gibson et al., 2007 \\
\hline 0.862 & 42.675 & 1880 & & & 32 & 2.7 & & & & & Zone 3 & Axial Zone & S-M & $1 / 24$ & Gibson et al., 2007 \\
\hline 0.867 & 42.675 & 1555 & & & 29 & 1.6 & & & & & Zone 3 & Axial Zone & S-M & $1 / 23$ & Gibson et al., 2007 \\
\hline
\end{tabular}

* S-S = Single sample, single grain; S-M = Single sample, multiple grain; M-M = Multiple sample, multiple grain; $M-S=$ Multiple sample, single grain; NR = Not Reported 


\begin{tabular}{|c|c|c|c|c|c|c|c|c|c|c|c|c|c|c|c|}
\hline Longitude & Latitude & $\begin{array}{l}\text { Elevation } \\
\text { (meters) }\end{array}$ & $\mathrm{AHe}$ & $\begin{array}{l}\mathrm{AHe} \\
\text { error }\end{array}$ & AFT & $\begin{array}{l}\text { AFT } \\
\text { error }\end{array}$ & ZHe & $\begin{array}{l}\text { ZHe } \\
\text { error }\end{array}$ & ZFT & $\begin{array}{l}\text { ZFT } \\
\text { error }\end{array}$ & $\begin{array}{c}\text { EW } \\
\text { Zone }\end{array}$ & Region & Sample type* & $\begin{array}{l}\text { \# Samples / } \\
\text { \# Grains }\end{array}$ & Reference \\
\hline 0.872 & 42.677 & 1315 & & & 30 & 1.9 & & & & & Zone 3 & Axial Zone & S-M & $1 / 22$ & Gibson et al., 2007 \\
\hline 0.679 & 42.458 & 1250 & & & 26 & 3.1 & & & & & Zone 3 & Axial Zone & S-M & $1 / 25$ & Gibson et al., 2007 \\
\hline 0.846 & 42.414 & 1490 & & & 30 & 2.7 & & & & & Zone 3 & Axial Zone & S-M & $1 / 24$ & Gibson et al., 2007 \\
\hline 0.851 & 42.413 & 1580 & & & 26 & 3.6 & & & & & Zone 3 & Axial Zone & S-M & $1 / 25$ & Gibson et al., 2007 \\
\hline 0.92 & 42.398 & 1540 & & & 30 & 4.1 & & & & & Zone 3 & Axial Zone & S-M & $1 / 39$ & Gibson et al., 2007 \\
\hline 0.921 & 42.402 & 1490 & & & 28 & 3.7 & & & & & Zone 3 & Axial Zone & S-M & $1 / 40$ & Gibson et al., 2007 \\
\hline 1.980 & 42.711 & 2190 & & & 55 & 4.4 & & & & & Zone 4 & Axial Zone & S-M & $1 / 20$ & Gunnel et al., 2009 \\
\hline 1.705 & 42.715 & 1939 & & & 41 & 1.7 & & & & & Zone 4 & Axial Zone & S-M & $1 / 20$ & Gunnel et al., 2009 \\
\hline 1.618 & 42.785 & 1850 & & & 50 & 2.5 & & & & & Zone 4 & Axial Zone & S-M & $1 / 20$ & Gunnel et al., 2009 \\
\hline 1.423 & 42.772 & 1938 & & & 59 & 4.7 & & & & & Zone 4 & Axial Zone & S-M & $1 / 20$ & Gunnel et al., 2009 \\
\hline 1.763 & 42.488 & 2900 & & & 50 & 3.2 & & & & & Zone 4 & Axial Zone & S-M & $1 / 18$ & Gunnel et al., 2009 \\
\hline 1.763 & 42.488 & 2900 & & & 51 & 3.8 & & & & & Zone 4 & Axial Zone & S-M & $1 / 20$ & Gunnel et al., 2009 \\
\hline 1.862 & 42.521 & 2377 & & & 35 & 2.5 & & & & & Zone 4 & Axial Zone & S-M & $1 / 11$ & Gunnel et al., 2009 \\
\hline 1.666 & 42.43 & 1950 & & & 33 & 1.8 & & & & & Zone 4 & Axial Zone & S-M & $1 / 19$ & Gunnel et al., 2009 \\
\hline-0.211 & 42.784 & 2449 & & & 23 & 1.6 & & & & & Zone 1 & Axial Zone & NR & NR & Herman et al., 2013 \\
\hline 0.165 & 42.824 & 1977 & & & 19 & 1.3 & & & & & Zone 2 & Axial Zone & NR & NR & Herman et al., 2013 \\
\hline 0.927 & 42.768 & 2490 & & & 17 & 4.5 & & & & & Zone 3 & Axial Zone & NR & NR & Herman et al., 2013 \\
\hline 1.862 & 42.703 & 1122 & & & 40 & 4.2 & & & & & Zone 4 & Axial Zone & NR & NR & Herman et al., 2013 \\
\hline 1.844 & 42.689 & 1331 & & & 31 & 1.5 & & & & & Zone 4 & Axial Zone & NR & NR & Herman et al., 2013 \\
\hline 0.128 & 42.959 & 88 & & & 36 & 2.5 & & & & & Zone 2 & Axial Zone & S-M & $1 / 16$ & Morris et al., 1998; Yelland, 1991 \\
\hline 0.22 & 42.716 & 1020 & & & 16 & 1.5 & & & & & Zone 2 & Axial Zone & S-M & $1 / 19$ & Morris et al., 1998; Yelland, 1991 \\
\hline 0.917 & 42.568 & 1635 & & & 36 & 3.4 & & & & & Zone 3 & Axial Zone & S-M & $1 / 15$ & Morris et al., 1998; Yelland, 1991 \\
\hline 0.844 & 42.5 & 1718 & & & 24 & 1.3 & & & & & Zone 3 & Axial Zone & S-M & $1 / 15$ & Morris et al., 1998; Yelland, 1991 \\
\hline 1.862 & 42.676 & 1000 & & & 27 & 1.3 & & & & & Zone 4 & Axial Zone & S-M & $1 / 10$ & Morris et al., 1998; Yelland, 1991 \\
\hline 1.789 & 42.649 & 1143 & & & 28 & 1.7 & & & & & Zone 4 & Axial Zone & S-M & $1 / 20$ & Morris et al., 1998; Yelland, 1991 \\
\hline 1.752 & 42.716 & 935 & & & 32 & 1.1 & & & & & Zone 4 & Axial Zone & S-M & $1 / 20$ & Morris et al., 1998; Yelland, 1991 \\
\hline 1.862 & 42.689 & 900 & & & 35 & 1.8 & & & & & Zone 4 & Axial Zone & S-M & $1 / 12$ & Morris et al., 1998; Yelland, 1991 \\
\hline 1.716 & 42.73 & 1160 & & & 35 & 1.9 & & & & & Zone 4 & Axial Zone & S-M & $1 / 14$ & Morris et al., 1998; Yelland, 1991 \\
\hline 1.752 & 42.568 & 1980 & & & 28 & 1.5 & & & & & Zone 4 & Axial Zone & S-M & $1 / 15$ & Morris et al., 1998; Yelland, 1991 \\
\hline-0.468 & 42.851 & 1660 & & & 21 & 1.5 & & & & & Zone 1 & Axial Zone & S-M & NR & Morris et al., 1998; Yelland, 1991 \\
\hline-0.193 & 42.77 & 2693 & & & 22 & 1.5 & & & & & Zone 1 & Axial Zone & S-M & NR & Morris et al., 1998; Yelland, 1991 \\
\hline 0.147 & 42.973 & 1645 & & & 38 & 3.0 & & & & & Zone 2 & Axial Zone & S-M & NR & Morris et al., 1998; Yelland, 1991 \\
\hline 0.385 & 42.878 & 700 & & & 35 & 2.8 & & & & & Zone 2 & Axial Zone & S-M & NR & Morris et al., 1998; Yelland, 1991 \\
\hline 0.11 & 42.838 & 2110 & & & 24 & 1.6 & & & & & Zone 2 & Axial Zone & S-M & NR & Morris et al., 1998; Yelland, 1991 \\
\hline 0.12 & 42.858 & 2321 & & & 27 & 1.8 & & & & & Zone 2 & Axial Zone & S-M & NR & Morris et al., 1998; Yelland, 1991 \\
\hline 0.147 & 42.811 & 2227 & & & 28 & 1.9 & & & & & Zone 2 & Axial Zone & S-M & NR & Morris et al., 1998; Yelland, 1991 \\
\hline 0.899 & 42.676 & 2104 & & & 27 & 1.7 & & & & & Zone 3 & Axial Zone & S-M & NR & Morris et al., 1998; Yelland, 1991 \\
\hline 0.734 & 42.541 & 1141 & & & 26 & 2.9 & & & & & Zone 3 & Axial Zone & S-M & NR & Morris et al., 1998; Yelland, 1991 \\
\hline 0.752 & 42.541 & 1631 & & & 30 & 3.5 & & & & & Zone 3 & Axial Zone & S-M & NR & Morris et al., 1998; Yelland, 1991 \\
\hline 1.767 & 42.664 & 1812 & & & 42 & 2.2 & & & & & Zone 4 & Axial Zone & S-M & NR & Morris et al., 1998; Yelland, 1991 \\
\hline 1.826 & 42.581 & 2353 & & & 24 & 1.3 & & & & & Zone 4 & Axial Zone & S-M & NR & Morris et al., 1998; Yelland, 1991 \\
\hline 1.807 & 42.514 & 1852 & & & 38 & 2.2 & & & & & Zone 4 & Axial Zone & S-M & NR & Morris et al., 1998; Yelland, 1991 \\
\hline-0.267 & 42.886 & 1417 & & & & & 26 & 6.5 & & & Zone 1 & Axial Zone & S-M & $1 / 4$ & Bosch et al., 2016 \\
\hline-0.29 & 42.839 & 3147 & & & & & 22 & 1.2 & & & Zone 1 & Axial Zone & S-M & $1 / 3$ & Bosch et al., 2016 \\
\hline-0.29 & 42.858 & 2080 & & & & & 22 & 1.7 & & & Zone 1 & Axial Zone & S-M & $1 / 3$ & Bosch et al., 2016 \\
\hline-0.239 & 42.754 & 1614 & & & & & 24 & 2.3 & & & Zone 1 & Axial Zone & S-M & $1 / 2$ & Bosch et al., 2016 \\
\hline-0.424 & 42.887 & 1066 & & & & & 26 & 2.0 & & & Zone 1 & Axial Zone & S-M & $1 / 3$ & Bosch et al., 2016 \\
\hline-0.41 & 42.91 & 1208 & & & & & 23 & 1.8 & & & Zone 1 & Axial Zone & S-M & $1 / 3$ & Bosch et al., 2016 \\
\hline-0.44 & 42.953 & 677 & & & & & 22 & 1.7 & & & Zone 1 & Axial Zone & S-M & $1 / 3$ & Bosch et al., 2016 \\
\hline-0.408 & 43.018 & 559 & & & & & 28 & 2.2 & & & Zone 1 & Axial Zone & S-M & $1 / 2$ & Bosch et al., 2016 \\
\hline 0.11 & 42.8 & 2879 & & & & & 29 & 1.7 & & & Zone 2 & Axial Zone & S-M & $1 / 8$ & Pik et al., 2016 \\
\hline 0.11 & 42.8 & 2754 & & & & & 25 & 1.5 & & & Zone 2 & Axial Zone & S-M & $1 / 6$ & Pik et al., 2016 \\
\hline 0.11 & 42.81 & 2707 & & & & & 29 & 1.8 & & & Zone 2 & Axial Zone & S-M & $1 / 15$ & Pik et al., 2016 \\
\hline 0.12 & 42.81 & 2423 & & & & & 29 & 1.7 & & & Zone 2 & Axial Zone & S-M & $1 / 20$ & Pik et al., 2016 \\
\hline 0.15 & 42.82 & 2038 & & & & & 31 & 1.8 & & & Zone 2 & Axial Zone & S-M & $1 / 10$ & Pik et al., 2016 \\
\hline 0.17 & 42.82 & 1864 & & & & & 35 & 2.1 & & & Zone 2 & Axial Zone & S-M & $1 / 11$ & Pik et al., 2016 \\
\hline 0.21 & 42.68 & 1099 & & & & & 20 & 1.2 & & & Zone 2 & Axial Zone & S-M & $1 / 20$ & Pik et al., 2016 \\
\hline 0.22 & 42.64 & 895 & & & & & 18 & 1.1 & & & Zone 2 & Axial Zone & S-M & $1 / 26$ & Pik et al., 2016 \\
\hline 0.23 & 42.67 & 1309 & & & & & 22 & 1.3 & & & Zone 2 & Axial Zone & S-M & $1 / 15$ & Pik et al., 2016 \\
\hline 0.25 & 42.68 & 1532 & & & & & 27 & 1.6 & & & Zone 2 & Axial Zone & S-M & $1 / 24$ & Pik et al., 2016 \\
\hline 0.27 & 42.66 & 2528 & & & & & 35 & 2.1 & & & Zone 2 & Axial Zone & S-M & $1 / 20$ & Pik et al., 2016 \\
\hline 0.27 & 42.68 & 1831 & & & & & 21 & 1.3 & & & Zone 2 & Axial Zone & S-M & $1 / 3$ & Pik et al., 2016 \\
\hline
\end{tabular}

* S-S = Single sample, single grain; S-M = Single sample, multiple grain; M-M = Multiple sample, multiple grain; M-S = Multiple sample, single grain; NR = Not Reported 


\begin{tabular}{|c|c|c|c|c|c|c|c|c|c|c|c|c|c|c|c|}
\hline 0.28 & 42.67 & 2221 & & & & & 41 & 2.5 & & & Zone 2 & Axial Zone & S-M & $1 / 19$ & Pik et al., 2016 \\
\hline 0.29 & 42.67 & 2209 & & & & & 38 & 2.3 & & & Zone 2 & Axial Zone & S-M & $1 / 13$ & Pik et al., 2016 \\
\hline 1.968 & 42.574 & 2320 & & & & & 46 & 3.7 & & & Zone 4 & Axial Zone & S-M & $1 / 2$ & Maurel et al., 2008 \\
\hline-0.226 & 42.77 & 2449 & & & & & & & 41 & 4.1 & Zone 1 & Axial Zone & S-M & NR & Yelland, 1991 (PhD thesis) \\
\hline 0.08 & 42.85 & 2700 & & & & & & & 33 & 3.3 & Zone 2 & Axial Zone & S-M & NR & Yelland, 1991 (PhD thesis) \\
\hline 0.15 & 42.84 & 2700 & & & & & & & 35 & 3.5 & Zone 2 & Axial Zone & S-M & NR & Yelland, 1991 (PhD thesis) \\
\hline 0.111 & 42.805 & 2707 & & & & & & & 46 & 2.5 & Zone 2 & Axial Zone & S-M & $1 / 24$ & Fillon et al., in review \\
\hline 0.119 & 42.808 & 2423 & & & & & & & 45 & 2.3 & Zone 2 & Axial Zone & S-M & $1 / 23$ & Fillon et al., in review \\
\hline 0.17 & 42.823 & 1864 & & & & & & & 46 & 2.0 & Zone 2 & Axial Zone & S-M & $1 / 48$ & Fillon et al., in review \\
\hline 0.993 & 42.714 & 2635 & & & & & & & 50 & 3.1 & Zone 3 & Axial Zone & S-M & $1 / 20$ & Sinclair et al., 2005 \\
\hline 0.99 & 42.507 & 1750 & & & & & & & 49 & 2.6 & Zone 3 & Axial Zone & S-M & $1 / 20$ & Sinclair et al., 2005 \\
\hline 0.767 & 42.59 & 1700 & & & & & & & 32 & 3.2 & Zone 3 & Axial Zone & S-M & NR & Yelland, 1991 (PhD thesis) \\
\hline 0.901 & 42.59 & 1700 & & & & & & & 41 & 4.1 & Zone 3 & Axial Zone & S-M & NR & Yelland, 1991 (PhD thesis) \\
\hline 1.768 & 42.51 & 2000 & & & & & & & 36 & 3.6 & Zone 4 & Axial Zone & S-M & NR & Yelland, 1991 (PhD thesis) \\
\hline 1.818 & 42.49 & 2000 & & & & & & & 37 & 3.7 & Zone 4 & Axial Zone & S-M & NR & Yelland, 1991 (PhD thesis) \\
\hline 1.777 & 42.6 & 2000 & & & & & & & 43 & 4.3 & Zone 4 & Axial Zone & S-M & NR & Yelland, 1991 (PhD thesis) \\
\hline 1.792 & 42.59 & 2000 & & & & & & & 39 & 3.9 & Zone 4 & Axial Zone & S-M & NR & Yelland, 1991 (PhD thesis) \\
\hline 0.267 & 42.663 & 2528 & & & & & & & 161 & 16 & Zone 2 & Axial Zone & S-M & $1 / 15$ & Fillon et al., in review \\
\hline 0.247 & 42.675 & 1532 & & & & & & & 143 & 7.7 & Zone 2 & Axial Zone & S-M & $1 / 33$ & Fillon et al., in review \\
\hline 0.813 & 42.515 & 1150 & & & & & & & 104 & 7.0 & Zone 3 & Axial Zone & S-M & $1 / 16$ & Sinclair et al., 2005 \\
\hline 1.105 & 42.391 & 805 & & & & & & & 159 & 33 & Zone 3 & Axial Zone & S-M & $1 / 3$ & Sinclair et al., 2005 \\
\hline \multirow{2}{*}{\multicolumn{16}{|c|}{ NORTH PYRENEAN ZONE }} \\
\hline & & & & & & & & & & & & & & & \\
\hline Longitude & Latitude & $\begin{array}{l}\text { Elevation } \\
\text { (meters) }\end{array}$ & $\mathrm{AHe}$ & $\begin{array}{l}\text { AHe } \\
\text { error }\end{array}$ & AFT & $\begin{array}{l}\text { AFT } \\
\text { error }\end{array}$ & $\mathrm{ZHe}$ & $\begin{array}{l}\mathrm{ZHe} \\
\text { error }\end{array}$ & ZFT & $\begin{array}{l}\text { ZFT } \\
\text { error }\end{array}$ & $\begin{array}{l}\text { EW } \\
\text { Zone }\end{array}$ & Region & Sample type* & $\begin{array}{l}\text { \# Samples / } \\
\text { \# Grains }\end{array}$ & Reference \\
\hline 1.44 & 42.831 & 2199 & 43 & 3.5 & & & & & & & & NPZ & S-M & $1 / 4$ & Vacherat et al., 2016 \\
\hline 1.44 & 42.827 & 2068 & 34 & 2.7 & & & & & & & & NPZ & S-M & $1 / 4$ & Vacherat et al., 2016 \\
\hline 1.441 & 42.824 & 1915 & 40 & 3.2 & & & & & & & & NPZ & S-M & $1 / 5$ & Vacherat et al., 2016 \\
\hline 1.439 & 42.821 & 1772 & 38 & 3.0 & & & & & & & & NPZ & S-S & $1 / 1$ & Vacherat et al., 2016 \\
\hline 1.436 & 42.818 & 1700 & 33 & 2.7 & & & & & & & & NPZ & S-M & $1 / 4$ & Vacherat et al., 2016 \\
\hline 1.437 & 42.813 & 1598 & 51 & 4.1 & & & & & & & & NPZ & S-M & $1 / 3$ & Vacherat et al., 2016 \\
\hline 1.442 & 42.805 & 1459 & 36 & 2.9 & & & & & & & & NPZ & S-M & $1 / 4$ & Vacherat et al., 2016 \\
\hline 1.247 & 42.86 & 552 & 44 & 3.5 & & & & & & & & NPZ & S-M & $1 / 1$ & Vacherat et al., 2016 \\
\hline 1.177 & 42.934 & 451 & 35 & 2.8 & & & & & & & & NPZ & S-M & $1 / 4$ & Vacherat et al., 2016 \\
\hline 1.214 & 42.908 & 493 & 34 & 3.0 & & & & & & & & NPZ & S-M & $1 / 3$ & Vacherat et al., 2016 \\
\hline 1.533 & 42.965 & 488 & 47 & 4.4 & & & & & & & & NPZ & S-M & $1 / 3$ & Vacherat et al., 2016 \\
\hline 1.133 & 42.933 & 1048 & & & 55 & 3.0 & & & & & & NPZ & S-M & $1 / 25$ & Fitzgerald et al., 1999 \\
\hline 1.202 & 42.943 & 815 & & & 50 & 3.0 & & & & & & NPZ & S-M & $1 / 25$ & Fitzgerald et al., 1999 \\
\hline 1.214 & 42.933 & 950 & & & 49 & 3.0 & & & & & & NPZ & S-M & $1 / 24$ & Fitzgerald et al., 1999 \\
\hline 1.215 & 42.94 & 645 & & & 49 & 2.0 & & & & & & NPZ & S-M & $1 / 25$ & Fitzgerald et al., 1999 \\
\hline 1.202 & 42.878 & 500 & & & 34 & 1.6 & & & & & & NPZ & S-M & $1 / 17$ & Morris et al., 1998 \\
\hline 1.551 & 42.959 & 455 & & & 106 & 5.3 & & & & & & NPZ & S-M & $1 / 20$ & Morris et al., 1998 \\
\hline 1.312 & 42.838 & 945 & & & 38 & 1.9 & & & & & & NPZ & S-M & $1 / 25$ & Morris et al., 1998 \\
\hline 1.147 & 42.905 & 520 & & & 40 & 1.7 & & & & & & NPZ & S-M & $1 / 15$ & Morris et al., 1998 \\
\hline 1.422 & 42.811 & 1604 & & & 35 & 2.2 & & & & & & NPZ & NR & NR & Yelland, 1991 (PhD thesis) \\
\hline 0.073 & 43.068 & 645 & & & 42 & 2.4 & & & & & & NPZ & NR & NR & Yelland, 1991 (PhD thesis) \\
\hline 1.679 & 42.824 & 1113 & & & 43 & 3.0 & & & & & & NPZ & NR & NR & Yelland, 1991 (PhD thesis) \\
\hline 1.385 & 43.027 & 426 & & & 118 & 12 & & & & & & NPZ & NR & NR & Yelland, 1991 (PhD thesis) \\
\hline 0.375 & 42.982 & 626 & & & 35 & 2.3 & & & & & & NPZ & S-M & $1 / 21$ & Mouchene, 2016 (PhD thesis) \\
\hline 0.595 & 42.957 & 677 & & & 38 & 6.0 & & & & & & NPZ & S-M & $1 / 12$ & Mouchene, 2016 (PhD thesis) \\
\hline 1.440 & 42.831 & 2199 & & & 47 & 4.7 & & & & & & NPZ & S-M & $1 / 20$ & Vacherat et al., 2016 \\
\hline 1.440 & 42.827 & 2068 & & & 37 & 2.2 & & & & & & NPZ & S-M & $1 / 22$ & Vacherat et al., 2016 \\
\hline 1.441 & 42.824 & 1915 & & & 46 & 4.0 & & & & & & NPZ & S-M & $1 / 20$ & Vacherat et al., 2016 \\
\hline 1.439 & 42.821 & 1772 & & & 38 & 2.4 & & & & & & NPZ & S-M & $1 / 27$ & Vacherat et al., 2016 \\
\hline 1.436 & 42.818 & 1700 & & & 35 & 2.4 & & & & & & NPZ & S-M & $1 / 31$ & Vacherat et al., 2016 \\
\hline 1.437 & 42.813 & 1598 & & & 42 & 1.9 & & & & & & NPZ & S-M & $1 / 20$ & Vacherat et al., 2016 \\
\hline 1.941 & 42.805 & 1459 & & & 42 & 3.4 & & & & & & NPZ & S-M & $1 / 27$ & Vacherat et al., 2016 \\
\hline 1.247 & 42.860 & 552 & & & 39 & 1.9 & & & & & & NPZ & S-M & $1 / 20$ & Vacherat et al., 2016 \\
\hline 1.177 & 42.934 & 451 & & & 44 & 2.1 & & & & & & NPZ & S-M & $1 / 56$ & Vacherat et al., 2016 \\
\hline 1.214 & 42.908 & 493 & & & 39 & 1.8 & & & & & & NPZ & S-M & $1 / 31$ & Vacherat et al., 2016 \\
\hline 1.533 & 42.964 & 488 & & & 75 & 7.0 & & & & & & NPZ & S-M & $1 / 29$ & Vacherat et al., 2016 \\
\hline
\end{tabular}

* S-S = Single sample, single grain; S-M = Single sample, multiple grain; M-M = Multiple sample, multiple grain; M-S = Multiple sample, single grain; NR = Not Reported 


\begin{tabular}{|c|c|c|c|c|c|c|c|c|c|c|c|c|c|c|c|}
\hline Longitude & Latitude & $\begin{array}{l}\text { Elevation } \\
\text { (meters) }\end{array}$ & AHe & $\begin{array}{l}\text { AHe } \\
\text { error }\end{array}$ & AFT & $\begin{array}{l}\text { AFT } \\
\text { error }\end{array}$ & ZHe & $\begin{array}{l}\text { ZHe } \\
\text { error }\end{array}$ & ZFT & $\begin{array}{c}\text { ZFT } \\
\text { error }\end{array}$ & $\begin{array}{c}\text { EW } \\
\text { Zone }\end{array}$ & Region & Sample type* & $\begin{array}{l}\text { \# Samples / } \\
\text { \# Grains }\end{array}$ & Reference \\
\hline 1.44 & 42.831 & 2199 & & & & & 89 & 18 & & & & NPZ & S-M & $1 / 2$ & Vacherat et al., 2016 \\
\hline 1.44 & 42.827 & 2068 & & & & & 68 & 17 & & & & NPZ & S-M & $1 / 3$ & Vacherat et al., 2016 \\
\hline 1.441 & 42.824 & 1915 & & & & & 78 & 16 & & & & NPZ & S-M & $1 / 3$ & Vacherat et al., 2016 \\
\hline 1.439 & 42.821 & 1772 & & & & & 60 & 3.8 & & & & NPZ & S-M & $1 / 3$ & Vacherat et al., 2016 \\
\hline 1.436 & 42.818 & 1700 & & & & & 68 & 4.2 & & & & NPZ & S-M & $1 / 3$ & Vacherat et al., 2016 \\
\hline 1.247 & 42.86 & 552 & & & & & 35 & 16 & & & & NPZ & S-M & $1 / 2$ & Vacherat et al., 2016 \\
\hline 1.177 & 42.934 & 451 & & & & & 60 & 18 & & & & NPZ & S-M & $1 / 3$ & Vacherat et al., 2016 \\
\hline 1.214 & 42.908 & 493 & & & & & 43 & 7.0 & & & & NPZ & S-M & $1 / 2$ & Vacherat et al., 2016 \\
\hline 1.533 & 42.965 & 488 & & & & & 41 & 23 & & & & NPZ & S-M & $1 / 3$ & Vacherat et al., 2016 \\
\hline 1.396 & 42.908 & 981 & & & & & 55 & 15 & & & & NPZ & S-M & $1 / 3$ & Vacherat et al., 2016 \\
\hline 1.364 & 42.838 & 1500 & & & & & 36 & 5.8 & & & & NPZ & S-M & $1 / 3$ & Vacherat et al., 2016 \\
\hline-0.275 & 43.103 & 439 & & & & & 44 & 5.0 & & & & NPZ & S-M & $1 / 3$ & Bosch et al., 2016 \\
\hline-0.252 & 43.152 & 317 & & & & & 220 & 55 & & & & NPZ & S-M & $1 / 3$ & Bosch et al., 2016 \\
\hline-0.320 & 43.069 & 778 & & & & & 36 & 3.0 & & & & NPZ & S-M & $1 / 3$ & Bosch et al., 2016 \\
\hline-0.234 & 43.119 & 367 & & & & & 38 & 4.1 & & & & NPZ & S-M & $1 / 3$ & Bosch et al., 2016 \\
\hline-0.287 & 43.172 & 266 & & & & & 205 & 60 & & & & NPZ & S-M & $1 / 3$ & Bosch et al., 2016 \\
\hline-0.408 & 43.018 & 559 & & & & & 28 & 2.3 & & & & NPZ & S-M & $1 / 2$ & Bosch et al., 2016 \\
\hline 1.44 & 42.831 & 2199 & & & & & & & 153 & 18 & & NPZ & S-M & $1 / 9$ & Vacherat et al., 2016 \\
\hline 1.44 & 42.827 & 2068 & & & & & & & 106 & 7.9 & & NPZ & S-M & $1 / 19$ & Vacherat et al., 2016 \\
\hline 1.441 & 42.824 & 1915 & & & & & & & 109 & 7.4 & & NPZ & S-M & $1 / 27$ & Vacherat et al., 2016 \\
\hline 1.439 & 42.821 & 1772 & & & & & & & 101 & 5.7 & & NPZ & S-M & $1 / 32$ & Vacherat et al., 2016 \\
\hline 1.436 & 42.818 & 1700 & & & & & & & 105 & 5.7 & & NPZ & S-M & $1 / 38$ & Vacherat et al., 2016 \\
\hline 1.247 & 42.86 & 552 & & & & & & & 169 & 19 & & NPZ & S-M & $1 / 12$ & Vacherat et al., 2016 \\
\hline 1.177 & 42.934 & 451 & & & & & & & 156 & 10 & & NPZ & S-M & $1 / 27$ & Vacherat et al., 2016 \\
\hline 1.214 & 42.8745 & 493 & & & & & & & 135 & 14 & & NPZ & S-M & $1 / 8$ & Vacherat et al., 2016 \\
\hline 1.533 & 42.965 & 488 & & & & & & & 168 & 29 & & NPZ & S-M & $1 / 7$ & Vacherat et al., 2016 \\
\hline 1.396 & 42.908 & 981 & & & & & & & 90 & 5.9 & & NPZ & S-M & $1 / 26$ & Vacherat et al., 2016 \\
\hline
\end{tabular}

* S-S = Single sample, single grain; S-M = Single sample, multiple grain; M-M = Multiple sample, multiple grain; $M-S$ = Multiple sample, single grain; NR = Not Reported

Thermochronology references: (Fillon et al., in review; Yelland, 1991; Morris et al., 1998; Fitzgerald et al., 1999; Sinclair et al., 2005; Denèle, 2007; Gibson et al., 2007; Jolivet et al., 2007; Maurel et al., 2008; Gunnell et al., 2009; Metcalf et al., 2009; Herman et al., 2013; Bosch et al., 2016; Mouchené, 2016; Pik et al., 2016; Vacherat et al., 2016)

\begin{tabular}{|l|c|c|}
\hline Table DR3. Model parameters & & \\
\hline Parameter & Value & Unit \\
\hline Crustal density & 2800 & $\mathrm{~kg} / \mathrm{m}^{3}$ \\
\hline Mantle density & 3300 & $\mathrm{~kg} / \mathrm{m}^{3}$ \\
\hline Young's modulus & 10 & $\mathrm{~Pa}$ \\
\hline Poisson's ratio & 0.3 & \\
\hline Thermal diffusivity & 25 & $\mathrm{~km}^{2} / \mathrm{Myr}$ \\
\hline Crustal thickness & 45 & $\mathrm{~km}$ \\
\hline Basal crustal temperature & 810 & ${ }^{\circ} \mathrm{C}$ \\
\hline Sea level temperature & 10 & ${ }^{\circ} \mathrm{C}$ \\
\hline Atmospheric lapse rate & 4 & ${ }^{\circ} \mathrm{C} / \mathrm{km}$ \\
\hline Crustal heat production & 0.95 & $\boldsymbol{\mu W} / \mathrm{m}^{3}$ \\
\hline Elastic plate thickness & 20 & $\mathrm{~km}$ \\
\hline
\end{tabular}




\section{SUPPLEMENTAL REFERENCES}

Bosch, G. V., Teixell, A., Jolivet, M., Labaume, P., Stockli, D., Domènech, M., and Monié, P., 2016, Timing of Eocene-Miocene thrust activity in the Western Axial Zone and Chaînons Béarnais (westcentral Pyrenees) revealed by multi-method thermochronology: Comptes Rendus Geoscience, v. 348, p. 246-256, doi:10.1016/j.crte.2016.01.001.

Braun, J., 2003, Pecube: a new finite-element code to solve the 3D heat transport equation including the effects of a time-varying, finite amplitude surface topography: Computers \& Geosciences, v. 29, p. 787-794, doi:10.1016/S0098-3004(03)00052-9.

Curry, M.E., van der Beek, P., Huismans, R.S., Wolf, S.G., and Muñoz, J.-A., 2019, Evolving paleotopography and lithospheric flexure of the Pyrenean Orogen from 3D flexural modeling and basin analysis: Earth and Planetary Science Letters, v. 515, p. 26-37, doi:10.1016/j.eps1.2019.03.009.

Denèle, Y., 2007, Formation des dômes gneissiques hercyniens dans les Pyrénées : exemple du massif de l’Aston-Hospitalet [Ph.D. Thesis]: Université de Toulouse Paul Sabatier.

England, P., and Molnar, P., 1990, Surface uplift, uplift of rocks, and exhumation of rocks: Geology, v. 18, p. 1173-1177, doi:10.1130/0091-7613(1990)018<1173:SUUORA>2.3.CO;2.

Fillon, C., and van der Beek, P., 2012, Post-orogenic evolution of the southern Pyrenees: constraints from inverse thermo-kinematic modelling of low-temperature thermochronology data: Basin Research, v. 24, p. 418-436, doi:10.1111/j.1365-2117.2011.00533.x.

Fillon, C., Mouthereau, F., Calassou, S., Pik, R., Bellahsen, N., Gautheron, C., Stockli, D., Mouchene, M., and van der Beek, P., in review, Extensional control on contrasted post-orogenic exhumation of the Pyrenees and its foreland

Fitzgerald, P.G., Muñoz, J.A., Coney, P.J., and Baldwin, S.L., 1999, Asymmetric exhumation across the Pyrenean Orogen; implications for the tectonic evolution of a collisional orogen: Earth and Planetary Science Letters, v. 173, p. 157-170.

Gibson, M., Sinclair, H.D., Lynn, G.J., and Stuart, F.M., 2007, Late- to post-orogenic exhumation of the 
Central Pyrenees revealed through combined thermochronological data and modelling: Basin Research, v. 19, p. 323-334, doi:10.1111/j.1365-2117.2007.00333.x.

Glotzbach, C., van der Beek, P.A., and Spiegel, C., 2011, Episodic exhumation and relief growth in the Mont Blanc massif, Western Alps from numerical modelling of thermochronology data: Earth and Planetary Science Letters, v. 304, p. 417-430, doi:10.1016/j.eps1.2011.02.020.

Gunnell, Y., Calvet, M., Brichau, S., Carter, A., Aguilar, J.-P., and Zeyen, H., 2009, Low long-term erosion rates in high-energy mountain belts: Insights from thermo- and biochronology in the Eastern Pyrenees:, doi:10.1016/j.epsl.2008.12.004.

Herman, F., Seward, D., Valla, P.G., Carter, A., Kohn, B., Willett, S.D., and Ehlers, T.A., 2013, Worldwide acceleration of mountain erosion under a cooling climate: Nature, v. 504, p. 423-426, doi:10.1038/nature12877.

Jammes, S., Huismans, R.S., and Muñoz, J.A., 2014, Lateral variation in structural style of mountain building: controls of rheological and rift inheritance: Terra Nova, v. 26, p. 201-207, doi:10.1111/ter.12087.

Jolivet, M., Labaume, P., Monié, P., Brunel, M., Arnaud, N., and Campani, M., 2007, Thermochronology constraints for the propagation sequence of the south Pyrenean basement thrust system (FranceSpain): Tectonics, v. 26, doi:10.1029/2006TC002080.

Maurel, O., Moniè, P., Pik, R., Arnaud, N., Brunel, M., and Jolivet, M., 2008, The Meso-Cenozoic thermotectonic evolution of the Eastern Pyrenees: an 40Ar/39Ar fission track and (U-Th)/He thermochronological study of the Canigou and Mont-Louis massifs: International Journal of Earth Sciences, v. 97, p. 565-584, doi:10.1007/s00531-007-0179-x.

Metcalf, J.R., Fitzgerald, P.G., Baldwin, S.L., and Muñoz, J.-A., 2009, Thermochronology of a convergent orogen: Constraints on the timing of thrust faulting and subsequent exhumation of the Maladeta Pluton in the Central Pyrenean Axial Zone: Earth and Planetary Science Letters, v. 287, p. 488-503, doi:10.1016/j.epsl.2009.08.036.

Morris, R.G., Sinclair, H.D., and Yelland, A.J., 1998, Exhumation of the Pyrenean orogen: implications 
for sediment discharge: Basin Reseach, v. 10, p. 69-85, doi:10.1046/j.1365-2117.1998.00053.x.

Mouchené, M., 2016, Evolution post-orogenique du systeme couple piemont/bassin versant : le ḿegacone alluvial de Lannemezan et son bassin versant au Nord des Pyrenees [Ph.D. Thesis]: Université Grenoble Alpes.

Muñoz, J.A., 1992, Evolution of a continental collision belt: ECORS-Pyrenees crustal balanced crosssection, in McClay, K.R. ed., Thrust Tectonics, London, Chapman \& Hall, p. 235-246.

Pik, R., Zimmerman, L., Bellahsen, N., Vacherat, A., Ternois, S., Mouthereau, F., and Ford, M., 2016, Damages dependent sensitivity of Zircon (U-Th)/He ages to thermal processes: the case of Pyrenean samples, in Geophysical Research Abstracts, EGU General Assembly, doi: 10.24396/ORDAR-52.

Sambridge, M., 1999a, Geophysical inversion with a neighbourhood algorithm-I. Searching a parameter space: Geophysical Journal International, v. 138, p. 479-494, doi:10.1046/j.1365246X.1999.00876.x.

Sambridge, M., 1999b, Geophysical inversion with a neighbourhood algorithm-II. Appraising the ensemble: Geophysical Journal International, v. 138, p. 727-746, doi:10.1046/j.1365246x.1999.00900.x.

Sinclair, H.D., Gibson, M., Naylor, M., and Morris, R.G., 2005, Asymmetric growth of the Pyrenees revealed through measurement and modeling of orogenic fluxes: American Journal of Science , v. 305, p. 369-406, doi:10.2475/ajs.305.5.369.

Teixell, A., Labaume, P., and Lagabrielle, Y., 2016, The crustal evolution of the west-central Pyrenees revisited: Inferences from a new kinematic scenario: Comptes Rendus Geoscience, v. 348, p. 257267, doi:10.1016/j.crte.2015.10.010.

Vacherat, A. et al., 2016, Rift-to-collision transition recorded by tectonothermal evolution of the northern Pyrenees: Tectonics, v. 35, p. 907-933, doi:10.1002/2015TC004016.

Valla, P.G., Herman, F., van der Beek, P.A., and Braun, J., 2010, Inversion of thermochronological ageelevation profiles to extract independent estimates of denudation and relief history — I: Theory and 
conceptual model: Earth and Planetary Science Letters, v. 295, p. 511-522, doi:10.1016/j.epsl.2010.04.033.

Yelland, A.J., 1991, Thermo-tectonics of the Pyrenees and Provence from fission track studies [Ph.D. Thesis]: University of London. 